Georgia State University

ScholarWorks @ Georgia State University

\title{
Confirmation of a Four-Factor Structure of the Schizotypal Personality Questionnaire among Undergraduate Students
}

\author{
Michael T. Compton \\ Emory University \\ Sandra M. Goulding \\ Emory University \\ Roger Bakeman \\ Georgia State University, bakeman@gsu.edu \\ Erin B. McClure-Tone \\ Georgia State University, Department of Psychology, etone@gsu.edu
}

Follow this and additional works at: https://scholarworks.gsu.edu/psych_facpub

Part of the Psychology Commons

\section{Recommended Citation}

Compton, Michael T.; Goulding, Sandra M.; Bakeman, Roger; and McClure-Tone, Erin B., "Confirmation of a Four-Factor Structure of the Schizotypal Personality Questionnaire among Undergraduate Students" (2009). Psychology Faculty Publications. 129.

https://scholarworks.gsu.edu/psych_facpub/129

This Article is brought to you for free and open access by the Department of Psychology at ScholarWorks @ Georgia State University. It has been accepted for inclusion in Psychology Faculty Publications by an authorized administrator of ScholarWorks @ Georgia State University. For more information, please contact scholarworks@gsu.edu. 
Confirmation of a Four-Factor Structure of the Schizotypal Personality Questionnaire among Undergraduate Students

Michael T. Compton, M.D., M.P.H. ${ }^{\text {a }}$

Sandra M. Goulding, M.P.H. ${ }^{a}$

Roger Bakeman, Ph.D. ${ }^{\mathrm{b}}$

Erin McClure-Tone, Ph.D. ${ }^{b}$

${ }^{a}$ Emory University School of Medicine, Department of Psychiatry and Behavioral Sciences, Atlanta, Georgia, USA, 30322

${ }^{\mathrm{b}}$ Georgia State University, Department of Psychology, Atlanta, Georgia, USA, 30302

Corresponding Author

Michael T. Compton, M.D., M.P.H.

Emory University School of Medicine

Department of Psychiatry and Behavioral Sciences

49 Jesse Hill Jr. Drive, S.E., Room \#333

Atlanta, GA 30303

TEL: 404-778-1486 / FAX: 404-616-3241

E-MAIL: Michael.Compton@emory.edu 


\begin{abstract}
Objective Although several exploratory and confirmatory factor analyses have supported the initially proposed factor structure of the Schizotypal Personality Questionnaire (SPQ) in which its nine subscales are grouped into cognitive-perceptual, interpersonal, and disorganized domains, others have revealed different latent structures. This study determined the best-fitting factor structure from among five models that have been proposed in the literature, as well as five additional hierarchically related models.

Method Undergraduate college students $(n=825)$ completed the SPQ as well as the Perceptual Aberration Scale (PAS) and the Revised Social Anhedonia Scale (SAS). Confirmatory factor analyses involving the nine SPQ subscales were conducted using the Linear Structural Relations Program (LISREL 8.72).

Results The best fitting model was a previously described 4-factor model including cognitiveperceptual, paranoid, negative, and disorganized domains. Correlations between the derived SPQ domains and the PAS score ranged $r=.26-.39$, and correlations between the SPQ domains and the SAS ranged $r=.07-.41$.

Conclusions The present findings support a 4-factor model over the standard 3-factor model that is typically used to derive SPQ subscale scores. The four derived domains are minimally to moderately correlated with other measures of psychosis-proneness.
\end{abstract}

Key Words: Confirmatory factor analysis; Perceptual Aberration Scale; Psychometric properties; Revised Social Anhedonia Scale; Schizotypal Personality Questionnaire; Schizotypy 


\section{Introduction}

The study of schizotypy is of increasing interest to schizophrenia researchers given evidence that schizotypy and schizotypal personality disorder (SPD) relate phenotypically (Catts et al., 2000; Kendler et al., 1994; Siever et al., 1993) and genetically (Clementz et al., 1991; Kendler et al., 1995, Silverman et al., 1993) to schizophrenia. Ongoing research on schizotypy in non-clinical samples will deepen the field's understanding of this complex personality construct as a vulnerability marker, as an aspect of some cases of the schizophrenia prodrome, and as an indicator that can enhance genetic studies. Schizotypy, like the related constructs of psychosis-proneness and psychoticism, is multidimensional, comprising multiple complex behavioral phenotypes. Some research has sought to determine differential correlates of schizotypy dimensions, which generally reflect the major groups of schizophrenia symptoms (i.e., positive, negative, and disorganized; Andreasen et al., 1995; Liddle, 1987). Ongoing attention to the psychometric properties and factorial structure of instruments designed to measure various facets of schizotypy is crucial.

Numerous well established, self-administered scales have been developed to measure the multidimensional schizotypy construct. These include the Schizophrenism Scale (Nielsen and Petersen, 1976; Venables et al., 1990), the Schizotypal Personality Scale (Claridge and Broks, 1984), the Rust Inventory of Schizotypal Cognitions (Rust, 1987; 1988), the Oxford-Liverpool Inventory of Feelings and Experiences (Mason et al., 1995), and the Schizophrenia Proneness Scale of the Minnesota Multiphasic Personality Inventory-2 (Bolinskey et al., 2003), among others. Studies have suggested that the Psychosis Proneness Scales (PPS), developed by Loren and Jean Chapman and colleagues, may offer the most reliable and valid means of identifying individuals with elevated levels of schizotypy (Grove, 1982; Lenzenweger, 1994), despite not 
mapping directly onto the nine SPD criteria of the Diagnostic and Statistical Manual of Mental Disorders, Fourth Edition, Text Revision (DSM-IV-TR; American Psychiatric Association, 2000). These scales include: (1) the Physical Anhedonia Scale (Chapman et al., 1976), assessing deficits in sensory pleasures; (2) the Perceptual Aberration Scale (Chapman et al., 1978), tapping gross body-image distortions; (3) the Magical Ideation Scale (Eckblad and Chapman, 1983), investigating causal beliefs that the dominant culture considers invalid and magical; (4) the Impulsive Nonconformity Scale (Chapman et al., 1984), measuring inability to comply with societal norms, empathize, and restrain impulsivity and self-gratification; and (5) the Revised Social Anhedonia Scale (Chapman et al., 1976; Eckblad et al., 1982), examining indifference to others.

The Schizotypal Personality Questionnaire (SPQ; Raine, 1991) was developed to reflect the nine DSM criteria for SPD (ideas of reference, odd beliefs or magical thinking, unusual perceptual experiences, paranoid ideation/suspiciousness, excessive social anxiety, no close friends, constricted affect, odd or eccentric behavior, and odd speech). Several exploratory (i.e., not requiring a priori hypotheses about how indicators are related to underlying factors or even the number of factors; Kline, 2005) and confirmatory (i.e., based on a priori measurement models in which both the number of factors and their correspondence to the indicators is explicitly specified; Kline, 2005) factor analytic studies have supported the initially proposed SPQ factor structure, wherein its nine subscales group into three domains: cognitive-perceptual, interpersonal, and disorganized (Calkins et al., 2004; Chen et al., 1997; Claridge et al., 1996; Gruzelier et al., 1996; Raine et al., 1994; Reynolds et al., 2000; Rossi and Daneluzzo, 2002). However, more recent confirmatory factor studies have suggested that other models of the latent 
structure (i.e., the underlying, hidden groupings of items that are intrinsic to the measure, but not necessarily obvious), of the SPQ may fit better (Stefanis et al., 2004; Wuthrich and Bates, 2006). In an effort to provide more information about the factorial validity of SPQ scores, the present study used confirmatory factor analysis to determine whether or not the initially described factorial structure of the nine SPQ subscales is supported in a relatively large sample of undergraduate students in the southeastern United States. Examination of this measurement model is crucial, given that numerous studies derive cognitive-perceptual, interpersonal, and disorganized subscale scores based on this factorial structure. Further, the present study aimed to select the best-fitting factor structure from among five models proposed in the literature (Kendler et al., 1991; Raine et al., 1994; Seiver and Gunderson, 1983; Stefanis et al., 2004; Wuthrich and Bates, 2006), as well as five additional hierarchically related, or nested models (i.e., one is a subset of the other after trimming or modifying the initial model based on theoretical or empirical considerations; Kline, 2005). It should be noted that these 10 models do not represent an exhaustive offering of potential models; for example, Boyle and Baxter performed a series of factor analyses on the SPQ and found a 2-factor solution that separated positive and negative schizotypal traits, as well as 3- and 4-factor models that further subdivided the positive traits (Green et al., 2008). Ongoing research on the factor structure of the SPQ and other measures of schizotypy is crucial given the importance of the schizotypy construct for both personality and behavioral research, as well as psychosis-proneness and schizophrenia research.

\section{Methods}

\subsection{Participants}

Study participants included 825 undergraduate college students. The mean $( \pm$ standard deviation) age of participants was $20.1 \pm 1.7$ years. Over three-quarters $(637,77.2 \%)$ were 
female, and nearly half (376, 45.6\%) were single and not dating anyone regularly. Almost half (371, 45.0\%) self-identified as White/Caucasian, 251 (30.4\%) as Black/African American, 84 (10.2\%) as Asian American, and 119 (14.4\%) as of one or more other racial/ethnic groups. Nearly half (391, 47.4\%) reported being in their freshman year and 118 (14.3\%) identified psychology as their undergraduate major. More than one-fourth of the students (234, 28.5\%) endorsed a history of mental health treatment. Among these 234 students, many reported a history of mental health treatment related to depression, anxiety, behavioral problems, or family issues; specifically, 41 (17.5\%) reported having sought treatment for a depression-related problem (e.g., depression, grief counseling, post-partum depression); 35 (15.0\%) had sought treatment for an anxiety-related problem (e.g., anxiety, posttraumatic stress disorder); 20 (8.6\%) had sought treatment for a behavioral disorder or problem (e.g., attention-deficit/hyperactivity disorder, anger management, addiction); and 12 (5.1\%) had sought treatment for family-related problems (e.g., parental divorce, relationship counseling, family counseling). Others reported treatment for various other problems, or did not provide a reason for treatment.

\subsection{Procedures}

Individuals aged $\geq 18$ years were invited to participate via a recruitment statement on an online program used to manage the undergraduate research pool. Interested students reviewed an online informed consent form before proceeding to the survey, and then completed a set of confidential web-based questionnaires. Participating students received extra course credit, though student participation was not required in this or any other study. Automated data entry produced computerized survey data files for data cleaning and analysis. Data from surveys completed in less than 20 minutes were excluded given that completion of the survey was expected to require longer than this. Additionally, only data from respondents aged 18-26 years 
were included in the analysis because the few older patients may not be typical of an undergraduate population.

\subsection{Measures}

The 74-item Schizotypal Personality Questionnaire (SPQ, Raine, 1991; Raine et al., 1994) was designed to address all nine DSM diagnostic criteria for SPD. Each “yes” response counts one point, with total scores ranging $0-74$. Items are grouped into nine subscales reflecting the DSM SPD criteria. As shown in Table 1, Cronbach's ( $\alpha$ ) internal consistency reliability coefficients for these subscales ranged .70-.83 (mean=.75) in the present sample, exceeding the means of .65 and .69 reported by Chen et al. (1997) for adults and adolescents, respectively. Scores to measure three domains of schizotypy are typically derived by simple summation of subscale scores: the cognitive-perceptual domain (ideas of reference, odd beliefs or magical thinking, unusual perceptual experiences, and paranoid ideation/suspiciousness subscales); the interpersonal domain (excessive social anxiety, no close friends, constricted affect, and paranoid ideation/suspiciousness subscales); and the disorganized domain (odd or eccentric behavior and odd speech subscales). Both exploratory (Calkins et al., 2004; Gruzelier et al., 1996) and confirmatory (Chen et al., 1997; Raine et al., 1994; Reynolds et al., 2000) factor analyses have suggested that the SPQ comprises these three factors. However, recent studies have demonstrated other factor structures (Stefanis et al., 2004; Wuthrich and Bates, 2006).

Two other traditional schizotypy scales were administered so that their correlations with the derived SPQ domains could be examined. The 35-item Perceptual Aberration Scale (PAS; Chapman et al., 1978) is a true/false, self-report measure designed to operationalize body-image distortions and perceptual anomalies (Chapman et al., 1978; Meehl, 1964; Meehl, 1990; Rado, 1960). Extensive past research demonstrates that the PAS is a well validated indicator of traits 
associated with schizotypy in both clinical and non-clinical populations (Champan et al., 1995;

Lenzenweger, 1998). The $\alpha$ coefficient for the PAS was .87 in the present sample, similar to the .88 reported by Kwapil et al. (2008) in 6,137 undergraduate students.

The 40-item Revised Social Anhedonia Scale (SAS; Chapman et al., 1976; Eckblad et al., 1982) is a true/false, self-report measure that assesses deficits in the ability to experience pleasure from interpersonal interactions. The SAS has been used extensively in clinical and nonclinical populations, has shown good reliability, appears to be relatively independent of other measures of psychosis-proneness (including the PAS), and identifies individuals exhibiting significant social maladjustment (Chapman and Chapman, 1985; Merritt et al., 1993). In the present sample, the $\alpha$ coefficient for the SAS was .86 , similar to the .84 reported by Kwapil et al. (2008) in their large sample of undergraduates.

\subsection{Data Analysis}

Confirmatory factor analyses were conducted using the Linear Structural Relations Program (LISREL 8.72) to examine the factorial structure of the SPQ. Specifically, five models proposed in the literature (Kendler et al., 1991; Raine et al., 1994; Seiver and Gunderson, 1983; Stefanis et al., 2004; Wuthrich and Bates, 2006), depicted in Figure 1, as well as five models hierarchically related to several of these, were examined. Several indices were selected a priori to assess the fit of measurement models to the data. First, based on the normal theory weighted least squares chi-square, the normed model chi-square is reported $\left(\chi^{2}{ }_{M} / \mathrm{df}_{\mathrm{M}}\right)$. Smaller values of the overall model chi-square $\left(\chi^{2}{ }_{M}\right)$ indicate goodness-of-fit (with $p>.05$ suggesting that the null hypothesis that the model fits the data cannot be rejected). The normed $\chi^{2}$ м partly reduces the sensitivity of $\chi^{2}$ м to sample size. Generally, values $<3.0$ indicate good fit. Second, the SteigerLind root mean square error of approximation (RMSEA) and its 90\% confidence interval (CI) 
provide a correction for model complexity. Small values are desired, with values $\geq .10$ indicating poor fit. The $90 \%$ CI of the RMSEA generally should not include .10. Third, the standardized root mean square residual (SRMR) assesses the mean absolute correlation residual. SRMR values $<.10$ are considered acceptable. Fourth, Bentler’s comparative fit index (CFI), ranging 0 1 , depends on the average size of the correlations in the data. The CFI is recommended to be $>.90$.

To compare hierarchical (nested) models, the chi-square difference $\left(\chi^{2}\right)$ test was used, in which the $\chi^{2}{ }_{\mathrm{M}}$ for the trimmed model is subtracted from that of the initial model, and the resulting value is divided by the difference in degrees of freedom $(d f)$. A non-significant value indicates approximately equal fit when comparing the two models (suggesting that the simpler model has not been over-simplified), and the more parsimonious model is preferred. To compare alternative factor solutions that are not hierarchically related, the Akaike information criterion (AIC), which favors more parsimonious models, is reported. When comparing two competing models, the one with the lowest AIC value is preferred.

Inter-correlations among the derived SPQ domains and PAS and SAS scores were examined using SPSS 15.0, as were internal consistency reliability coefficients.

\section{Results}

Fit indices for the ten measurement models are given in Table 2. The first model is the 4-factor "paranoid" model of Stefanis et al. (2004). As depicted in Figure 1A, this is a multidimensional model (i.e., one or more indicators load on more than one factor) in that the paranoid ideation/suspiciousness and excessive social anxiety subscales load on both the paranoid and negative factors. This model fit the data well (Table 2). Two modifications of this well-fitting model were examined, one in which the paranoid ideation/suspiciousness subscale, 
but not the excessive social anxiety subscale, loads on both the paranoid and negative factors; and the other in which the excessive social anxiety, but not the paranoid ideation/suspiciousness subscale, loads on both the paranoid and negative factors. Neither of these models fit as well as the first. A unidimensional modification of the first model also was examined, in which the paranoid factor includes the ideas of reference and paranoid ideation/suspiciousness subscales, and the negative factor includes the excessive social anxiety, no close friends, and constricted affect subscales. Again, this model did not fit as well as the first model put forth by Stefanis et al. (2004). Thus, of the 4-factor models examined, the best fit (all indices, including the normed chi-square, falling within acceptable ranges) occurred when the paranoid ideation/suspiciousness subscale and the excessive social anxiety subscale load on both paranoid and negative factors. The 4-factor model with the paranoid ideation/suspiciousness subscale loading on both the paranoid and negative factors fit next best, but significantly worse (e.g., the normed chi-square was 4.4, which is greater than the conventional standard of 3.0).

Three 3-factor models were assessed. As shown in Figure 1B, the modified 3-factor model of Wuthrich and Bates (2006) is multidimensional in that three subscales (odd beliefs or magical thinking, paranoid ideation/suspiciousness, and excessive social anxiety) load on both the cognitive-perceptual and interpersonal factors. Neither this model nor the standard 3-factor model of Raine et al. (1994) (see Figure 1C) — used by many researchers to derive subscale scores for cognitive-perceptual, interpersonal, and disorganized domains—-fit the data adequately (Table 2). A unidimensional modification of the Raine et al. (1994) model, in which the cognitive-perceptual factor includes the odd beliefs or magical thinking subscale and the unusual perceptual experiences subscale, and the interpersonal factor includes the ideas of reference, paranoid ideation/suspiciousness, excessive social anxiety, no close friends, and 
constricted affect subscales, also did not fit the data (Table 2). Thus, this latter unidimensional 3-factor model clearly does not fit well; the other 3-factor models fit better, but none have values of RMSEA or the normed chi-square that were within acceptable ranges.

Two 2-factor models were examined: the 2-factor model suggested by conceptualizations of Kendler et al. (1991) (Figure 1D), and the simple 2-factor model suggested by descriptions of schizotypy by Siever and Gunderson (1983) (Figure 1E). Neither of these models fit the data, as indicated by the fit statistics (Table 2). Finally, a very simple model in which all nine subscales load onto one factor was assessed. Again, this model did not fit the data.

Although the SRMR was $<.10$ for all models, the upper 90\% CI of the RMSEA was $<.10$ and the normed chi-square was $<3.0$ only for the Stefanis et al. (2004) 4-factor model. In particular, the standard 3-factor model of Raine et al. (1994) fit significantly worse than this 4factor model, to which it relates hierarchically $\left(\chi^{2}{ }_{\mathrm{D}}(4, N=825)=247, p<.001\right)$.

Internal consistency reliability coefficients for domain scores derived using the best fitting 4-factor model ranged .81-.89 (Table 3). As documented in a prior report on schizotypy and substance use in this sample (Esterberg et al., 2009), mean ( \pm standard deviation) scores for the cognitive-perceptual, paranoid, negative, and disorganized subscales were 3.9 $\pm 3.4,9.8 \pm 5.6$, 9.9 \pm 6.8 , and 5.3 \pm 4.1 , respectively. Correlations between the SPQ total score and the PAS and SAS were $r=.38$ and $r=.30$, respectively, and the correlation between PAS and SAS scores was $r=.21$. Inter-correlations between the SPQ domain scores, PAS score, and SAS score are shown in Table 3. Also, as previously reported (Esterberg et al., 2009), correlations between the four SPQ domains were quite high ( $r=.43-.84)$, though it should be noted that the highest correlation is largely driven by overlap among two subscales (paranoid ideation/suspiciousness and excessive social anxiety) in the paranoid and negative domains (the only overlapping domains). 
Correlations between the derived SPQ domains and the PAS ranged $r=.26-.39$, and correlations between the SPQ domains and the SAS ranged $r=.07-.41$.

\section{Discussion}

Several key findings emerged from this analysis. First, of the 10 models tested, the 4factor model introduced by Stefanis et al. (2004) in a study of 1,355 young male conscripts in the Greek Air Force provided the best fit to the data. This suggests that in the present sample, and perhaps others, subscale scores derived from this structural model may have greater factorial validity than those more commonly used in schizotypy research in recent years (i.e., the cognitive-perceptual, interpersonal, and disorganized domains based on the initial conceptualization and factor analyses of Raine and colleagues (Raine, 1991; Raine et al., 1994)). Although Raine et al. (1994) found support for the 3-factor model among 822 undergraduate students and other studies have confirmed this latent structure (Chen et al., 1997; Claridge et al., 1996; Reynolds et al., 2000; Rossi and Daneluzzo, 2002), the 4-factor model that included a paranoid factor evidently was not tested. At their initial demonstration of the 3-factor model, the authors recommended further testing to assess the model’s factorial validity (Raine et al., 1994). Other studies have confirmed that simpler 1-factor and 2-factor models do not provide good fit to the data (Chen et al., 1997; Raine et al., 1994; Reynolds et al., 2000; Stefanis et al., 2004; Wuthrich and Bates, 2006). Although it could be suggested that the present findings supporting the 4-factor model of Stefanis et al. (2004) are due to close similarities between the current sample and theirs, this is an unlikely sole explanation given that other factor analytic studies (e.g., Raine et al., 1994; Wuthrich and Bates, 2006) were conducted with English-speaking undergraduate samples similar to the one used in this study. Future large-sample studies of schizotypy and its correlates should conduct similar confirmatory factor analyses before 
necessarily relying on the standard cognitive-perceptual, interpersonal, and disorganized subscales.

A second key finding was that correlations among derived SPQ domains (based on the best-fitting 4-factor model) were generally moderate (.43-.58), except for the high correlation between the paranoid and negative domains (.84), which is expected given that two subscales overlap in these domains. Correlations between the derived SPQ domains and the PAS and SAS were generally low (.07-41), which could indicate that the three instruments measure different aspects of schizotypy or that one or more of the measures do not validly measure the schizotypy construct. Of note, although anhedonia is central to some conceptualizations of schizotypy, this trait is largely absent from the SPQ because DSM criteria for SPD do not include this feature (Mason et al., 1997). This could account for the low correlations between scores on the derived SPQ domains and the SAS. The PAS and SAS were mildly correlated (.21), which is consistent with the correlation observed by Pope and Kwapil (2000) in 523 undergraduates (.32) and by Kwapil et al. (2008) in a combined sample of 6,137 undergraduates (.29). A third finding, as expected based on the confirmatory factor analysis, is that internal consistency reliabilities were acceptable for the four derived SPQ domains. It should be noted, however, that in addition to indicating internal consistency/item homogeneity, internal consistency reliability coefficients may also suggest a high level of item redundancy or the rephrasing of items in several different ways (Boyle, 1991).

Several methodological limitations of this study should be recognized. First, generalizability may be limited given that generally healthy, high-functioning, predominantly female undergraduates constituted the sample. Schizotypy scores have consistently been found to be higher in adolescents and young adults than in older adults (Raine, 2009). It is possible that 
the factorial structure of the SPQ varies by sample-specific characteristics, which could account for differences across past studies. However, total SPQ scores showed good variability and $28 \%$ of participants had a history of mental health treatment, indicating that the sample was not exceptionally or unusually healthy. That being said, it is likely that more than $28 \%$ of the sample may have had a history of a mental illness given the under-recognition and under-treatment of mental illnesses in the general population. Although it is assumed that the present sample is typical of a random undergraduate sample, this cannot be confirmed. Other sample-specific characteristics could have potentially influenced the findings. For example, the level of motivation to participate, and honesty or accuracy in reporting, could have been affected by the fact that respondents received extra credit and they were not interacting directly with a researcher (but rather completing an online survey). In an attempt to mitigate effects of low motivation or inaccuracy, data from surveys completed in less than 20 minutes were excluded given that completion of the survey was expected to require longer than this.

A second methodological limitation is that correlations between the derived SPQ domains and other measures of schizotypy were limited to PAS and SAS scores, and all three measures were self-report. Other domains of schizotypy, such as impulsive nonconformity, have been suggested as missing from the SPQ subscales (Gruzelier, 1996). Along these lines, the present study focused on the latent factorial structure of a measurement instrument and the identification of best-fitting models therefore reflects the nature of the measure administered (Kwapil et al., 2008), rather than the complex schizotypy construct itself. Third, other measurement models could have been tested (e.g., the 2-, 3-, and 4-factor models studied by Boyle and Baxter; Green et al., 2008). Furthermore, only select psychometric properties were 
examined, given that the focus was on factorial validity; data on test-retest reliability would have been beneficial in addition to internal consistency reliability.

In summary, the findings of the present study support the 4-factor model described by Stefanis et al. (2004) over those of Raine et al. (1994) and Wuthrich and Bates (2006). Furthermore, simpler 2-factor models based on earlier conceptualizations of the latent structure of schizotypy (Kendler et al., 1991; Siever and Gunderson, 1983), which also have been disconfirmed in prior studies (Chen et al., 1997; Raine et al., 1994; Reynolds et al., 2000; Stefanis et al., 2004; Wuthrich and Bates, 2006), did not fit the data from the present sample. Five other models that are hierarchically related to several of these also were not supported in the present sample. Research on schizotypy may benefit from using domain scores derived from the 4-factor model of the SPQ, though additional confirmatory factor analyses in other large samples is warranted to further clarify ideal derivations of SPQ domains. The reliable and valid measurement of the multi-dimensional schizotypy construct is critical to advancing understandings of psychological functioning in both general population groups and clinical samples. 


\section{References}

American Psychiatric Association, 2000. Diagnostic and Statistical Manual of Mental Disorders, Fourth Edition. Washington, DC.

Andreasen, N.C., Arndt, S., Alliger, R., Miller, D., Flaum, M., 1995. Symptoms of schizophrenia: methods, meanings, and mechanisms. Archives of General Psychiatry 52, $341-51$.

Bolinesky, P.K., Gottesman, I.I., Nichols, D.S., 2003. The Schizophrenia Proneness (SzP) Scale: An MMPI-2 measure of schizophrenia liability. Journal of Clinical Psychology 37, 107108.

Boyle, G.J., 1991. Does item homogeneity indicate internal consistency or item redundancy in psychometric scales? Personality and Individual Differences 12, 291-94.

Calkins, M.E., Curtis, C.E., Grove, W.M., Iacono, W.G., 2004. Multiple dimensions of schizotypy in first degree biological relatives of schizophrenia patients. Schizophrenia Bulletin 30, 317-325.

Catts, S.V., Fox, A.M., Ward, P.B., McConaghy, N., 2000. Schizotypy: phenotypic marker as risk factor. Australian and New Zealand Journal of Psychiatry 34, S101-107.

Chapman, J.P., Chapman, L.J., Kwapil, T.R., 1995. Scales for the measurement of schizotypy. In: Raine, A., Lencz, T., Mednick, S.A. (Eds.), Schizotypal Personality (pp. 79-106). New York: Cambridge University Press.

Chapman, L.J., Chapman, J.P., Raulin, M.L., 1976. Scales for physical and social anhedonia. Journal of Abnormal Psychology 85, 374-382. 
Chapman, L.J., Chapman, J.P., Numbers, J.S., Edell, W.S., Carpenter B.N., Beckfield, D., 1984. Impulsive nonconformity as a trait contributing to the prediction of psychoticlike and schizotypal symptoms. Journal of Nervous and Mental Disease 172, 681-691.

Chapman, L.J., Chapman, J.P., 1985. Psychosis proneness. In: Alpert, M. (Ed.), Controversies in Schizophrenia: Changes and Constancies (pp. 157-174). New York: Guilford.

Chapman, L.J., Chapman, J.P., Raulin, M.L., 1978. Body-image aberration in schizophrenia. Journal of Abnormal Psychology 87, 399-407.

Chen, W.J., Hsiao, C.K., Lin, C.C.H., 1997. Schizotypy in community samples: The three-factor structure and correlation with sustained attention. Journal of Abnormal Psychology 106, $649-654$.

Claridge, G., Broks, P., 1984. Schizotypy and hemisphere function - I: Theoretical considerations and the measurement of schizotypy. Personality and Individual Differences 5, 633-648.

Claridge, G., McCreery, C., Mason, E., Bentall, R., Boyle, G., Slade, P., Popplewell, D., 1996. The factor structure of "schizotypal” traits: A large replication study. British Journal of Clinical Psychology 35, 103-115.

Clementz, B.A., Grove, W.M., Katsanis, J., Iacono, W.G., 1991. Psychometric detection of schizotypy: Perceptual aberration and physical anhedonia in relatives of schizophrenics. Journal of Abnormal Psychology 100, 607-612.

Eckblad, M., Chapman, L.J., 1983. Magical ideation as an indicator of schizotypy. Journal of Consulting and Clinical Psychology 51, 215-225.

Eckblad, M.L., Chapman, L.J., Chapman, J.P., Mishlove, M., 1982. The Revised Social Anhedonia Scale. Unpublished test. University of Wisconsin-Madison. 
Esterberg, M.L., Goulding, S.M., McClure-Tone, E.B., Compton, M.T., 2009. Schizotypy and nicotine, alcohol, and cannabis use in a non-psychiatric sample. Addictive Behaviors 34, 374-379.

Green, M., Boyle, G.J., Raine, A., 2008. Schizotypal personality models. In G.J. Boyle, G. Matthews, D.H. Saklofske. (Eds.). Handbook of Personality Theory and Assessment: Vol. 1 - Personality Theories and Models (Chapter 19, pp. 399-419). Los Angeles: Sage Publishers.

Grove, W.M., 1982. Psychometric detection of schizotypy. Psychological Bulletin 92, 27-38.

Gruzelier, J.H., 1996. The factorial structure of schizotypy: Part I. Affinities with syndromes of schizophrenia. Schizophrenia Bulletin 22, 611-620.

Kendler, K.S., Ochs, A.L., Gorman, A.M., Hewitt, J.K., Ross, D.E., Mirsky, A.F., 1991. The structure of schizotypy: A pilot multitrait twin study. Psychiatry Research 36, 19-36.

Kendler, K.S., Gruenberg, A.M., Kinney, D.K., 1994. Independent diagnoses of adoptees and relatives as defined by DSM-III in the provincial and national samples of the Danish adoption study of schizophrenia. Archives of General Psychiatry 51, 456-468.

Kendler, K.S., McGuire, M., Gruenberg, A.M., Walsh, D., 1995. Schizotypal symptoms and signs in the Roscommon Family Study: Their factor structure and familial relationship with psychotic and affective disorders. Archives of General Psychiatry 52, 296-303.

Kline, R.B., 2005. Principles and Practice of Structural Equation Modeling. The Guilford Press. New York, NY.

Kwapil, T.R., Barrantes-Vidal, N., Silvia, P.J., 2008. The dimensional structure of the Wisconsin Schizotypy Scales: Factor identification and construct validity. Schizophrenia Bulletin 34, 444-457. 
Lenzenweger, M.F., 1994. Psychometric high-risk paradigm, perceptual aberrations, and schizotypy: An update. Schizophrenia Bulletin 20, 121-135.

Liddle, P.F., 1987. The symptoms of chronic schizophrenia: a re-examination of the positivenegative dichotomy. British Journal of Psychiatry 151, 145-151.

Lenzenweger, M.F., 1998. Schizotypy and schizotypic psychopathology: Mapping an alternative expression of schizophrenia liability. In: Lenzenweger, M.F., Dworkin, R.H. (Eds.), Origins and Development of Schizophrenia. Advances in Experimental Psychopathology (pp. 93-295). Washington, DC: American Psychological Association.

Mason, O., Claridge, G., Jackson, M., 1995. New scales for the assessment of schizotypy. Personality and Individual Differences 1, 7-13.

Mason, O., Claridge, G., Williams, L., 1997. Questionnaire measurement. In: Claridge, G. (Ed.), Schizotypy: Implications for Illness and Health (pp. 19-37). New York: Oxford University Press.

Meehl, P.E., 1964. Manual for the Use with Checklist of Schizotypic Signs. Unpublished manuscript, University of Minnesota.

Meehl, P.E., 1990. Toward an integrated theory of schizotaxia, schizotypy, and schizophrenia. Archives of General Psychiatry 46, 873-877.

Merritt, R.D., Balogh, D.W., DeVinney, S.E., 1993. Use of the MMPI to assess the construct validity of the revised Social Anhedonia Scale as an index of schizotypy. Journal of personality assessment 60, 227-238.

Nielsen, T.C., Petersen, N.E., 1976. Electrodermal correlates of extraversion, trait anxiety, and schizophrenism. Scandinavian Journal of Psychology 17, 73-80. 
Pope, C.A., Kwapil, T.R., 2000. Dissociative experiences in hypothetically psychosis-prone college students. Journal of Nervous and Mental Disease, 188, 530-536.

Rado, S., 1960. Theory and therapy: The theory of schizotypal organization and its application to the treatment of decompensated schizotypal behavior. In: Scher, S.C., Davis, H.R. (Eds.), The Outpatient Treatment of Schizophrenia (pp. 87-101). New York: Grune \& Stratton.

Raine, A., 2009. Manual for the Schizotypal Personality Questionnaire (SPQ and SPQ-B). http://www-rcf.usc.edu/'raine/ Accessed January 21, 2009.

Raine, A., 1991. The SPQ: A scale for the assessment of schizotypal personality based on DSMIII-R criteria. Schizophrenia Bulletin 17, 555-564.

Raine, A., Reynolds, C., Lencz, T., Scerbo, A., Triphon, N., Kim, D., 1994. Cognitiveperceptual, interpersonal, and disorganized features of schizotypal personality. Schizophrenia Bulletin 20, 191-201.

Reynolds, C.A., Raine, A., Mellingen, K., Venables, P.H., Mednick, S.A., 2000. Three-factor model of schizotypal personality: Invariance across culture, gender, religious affiliation, family adversity, and psychopathology. Schizophrenia Bulletin 26, 603-618

Rossi, A., Daneluzzo, E., 2002. Schizotypal dimensions in normals and schizophrenic patients: A comparison with other clinical samples. Schizophrenia Research 54, 67-75.

Rust, J., 1987. The Rust Inventory of Schizoid Cognitions (RISC): A psychometric measure of psychoticism in the normal population. British Journal of Clinical Psychology 26, 151152.

Rust, J., 1988. The Rust Inventory of Schizotypal Cognitions (RISC). Schizophrenia Bulletin 14, $317-322$. 
Siever, L.J., Gunderson, J.G., 1983. The research for a schizotypal personality: Historical origins and current status. Comprehensive Psychiatry 24, 199-212.

Siever, L.J., Kalus, O.F., Keefe, R.S.E., 1993. The boundaries of schizophrenia. Psychiatric Clinics of North America 16, 217-244.

Silverman, J.M., Siever, L.J., Horvath, T.B., Coccaro, E.F., Klar, H.M., Davidson, M., Pinkham, L., Apter, S.H., Mohs, R.C., Davis, K.L., 1993. Schizophrenia-related and affective personality disorder traits in relatives of probands with schizophrenia and personality disorders. American Journal of Psychiatry 150, 435-442.

Stefanis, N.C., Smyrnis N., Avramopoulos, D., Evdokimidis, I., Ntzoufras, I., Stefanis, C.N., 2004. Factorial composition of self-rated schizotypal traits among young males undergoing military training. Schizophrenia Bulletin 30, 335-350.

Venables, P.H., Wilkins, S., Mitchell, D.A., Raine, A., Bailes, K., 1990. A scale for the measurement of schizotypy. Personality and Individual Differences 11, 481-495.

Wuthrich, V.M., Bates, T.C., 2006. Confirmatory factor analysis of the three-factor structure of the Schizotypal Personality Questionnaire and Chapman schizotypy scales. Journal of Personality Assessment 87, 292-304. 


\section{Table 1. Internal Consistency Reliabilities (Cronbach's $\alpha$ ) of the SPQ Subscales}

\begin{tabular}{|l|c|c|}
\hline SPQ Subscale & Number of Items & $\boldsymbol{\alpha}$ \\
\hline Ideas of reference & 9 & .74 \\
\hline Odd beliefs or magical thinking & 7 & .70 \\
\hline Unusual perceptual experiences & 9 & .73 \\
\hline Paranoid ideation/suspiciousness & 8 & .75 \\
\hline Excessive social anxiety & 8 & .73 \\
\hline No close friends & 9 & .75 \\
\hline Constricted affect & 8 & .71 \\
\hline Odd or eccentric behavior & 7 & .83 \\
\hline Odd speech & 9 & .77 \\
\hline
\end{tabular}




\begin{tabular}{|c|c|c|c|c|c|c|c|}
\hline Model & $\chi_{M}^{2}$ & $d f_{M}$ & $\chi^{2}$ normed ${ }^{a}$ & RMSEA $(90 \%$ CI) & $S R M R$ & CFI & AIC \\
\hline $\begin{array}{l}\text { a modification of A. above, in which PI, but not ESA, } \\
\text { loads on both the paranoid and negative factors }\end{array}$ & 88 & 20 & 4.4 & $.064(.052, .078)$ & .032 & .98 & 128 \\
\hline $\begin{array}{l}\text { a modification of A. above, in which ESA, but not PI, } \\
\text { loads on both the paranoid and negative factors }\end{array}$ & 145 & 20 & 7.2 & $.087(.074, .10)$ & .037 & .95 & 195 \\
\hline $\begin{array}{l}\text { a unidimensional modification of A. above, in which } \\
\text { paranoid includes IOR and PI, and negative includes } \\
\text { ESA, NCF, and CA }\end{array}$ & 173 & 21 & 8.4 & $.094(.081, .11)$ & .042 & .95 & 221 \\
\hline C. the standard 3-factor model of Raine et al. (1994) & 296 & 23 & 12.9 & $.12(.11, .13)$ & .051 & .92 & 340 \\
\hline $\begin{array}{l}\text { a unidimensional modification of C. above, in which } \\
\text { cognitive-perceptual includes OBMT and UPE, and } \\
\text { interpersonal includes IOR, PI, ESA, NCF, and CA }\end{array}$ & 484 & 24 & 20.2 & $.15(.14, .15)$ & .073 & .87 & 526 \\
\hline D. the 2-factor model of Kendler et al. (1991) & 521 & 24 & 21.7 & $.16(.15, .17)$ & .092 & .84 & 526 \\
\hline E. the simple 2-factor model of Siever and Gunderson (1983) & 473 & 26 & 18.2 & $.14(.13, .16)$ & .070 & .87 & 511 \\
\hline $\begin{array}{l}\text { a modification of E. above, in which all nine subscales } \\
\text { load onto one single factor }\end{array}$ & 872 & 27 & 32.3 & $.19(.18, .21)$ & .088 & .77 & 908 \\
\hline
\end{tabular}


Table 3. Inter-correlations among the Four Derived SPQ Domains and PAS and SAS Scores, and Internal Consistency Reliability Coefficients (in Italics along the Diagonal)

\begin{tabular}{|l|l|l|l|l|l|l|}
\hline & SPQ-CP & SPQ-P & SPQ-N & SPQ-D & PAS & SAS \\
\hline SPQ Cognitive-Perceptual (16 items) & .81 & & & & & \\
\hline SPQ Paranoid (25 items) & $.52^{* *}$ & .86 & & & & \\
\hline SPQ Negative (33 items) & $.43^{* *}$ & $.84^{* *}$ & .89 & & & \\
\hline SPQ Disorganized (16 items) & $.55^{* *}$ & $.56^{* *}$ & $.58^{* *}$ & .86 & & \\
\hline PAS (35 items) & $.35^{* *}$ & $.26^{* *}$ & $.28^{* *}$ & $.39 * *$ & .87 & \\
\hline SAS (40 items) & $.07^{*}$ & $.21^{* *}$ & $.41^{* *}$ & $.19^{* *}$ & $.21^{* *}$ & .86 \\
\hline
\end{tabular}

$* p=.05$

$* * p<.001$ 


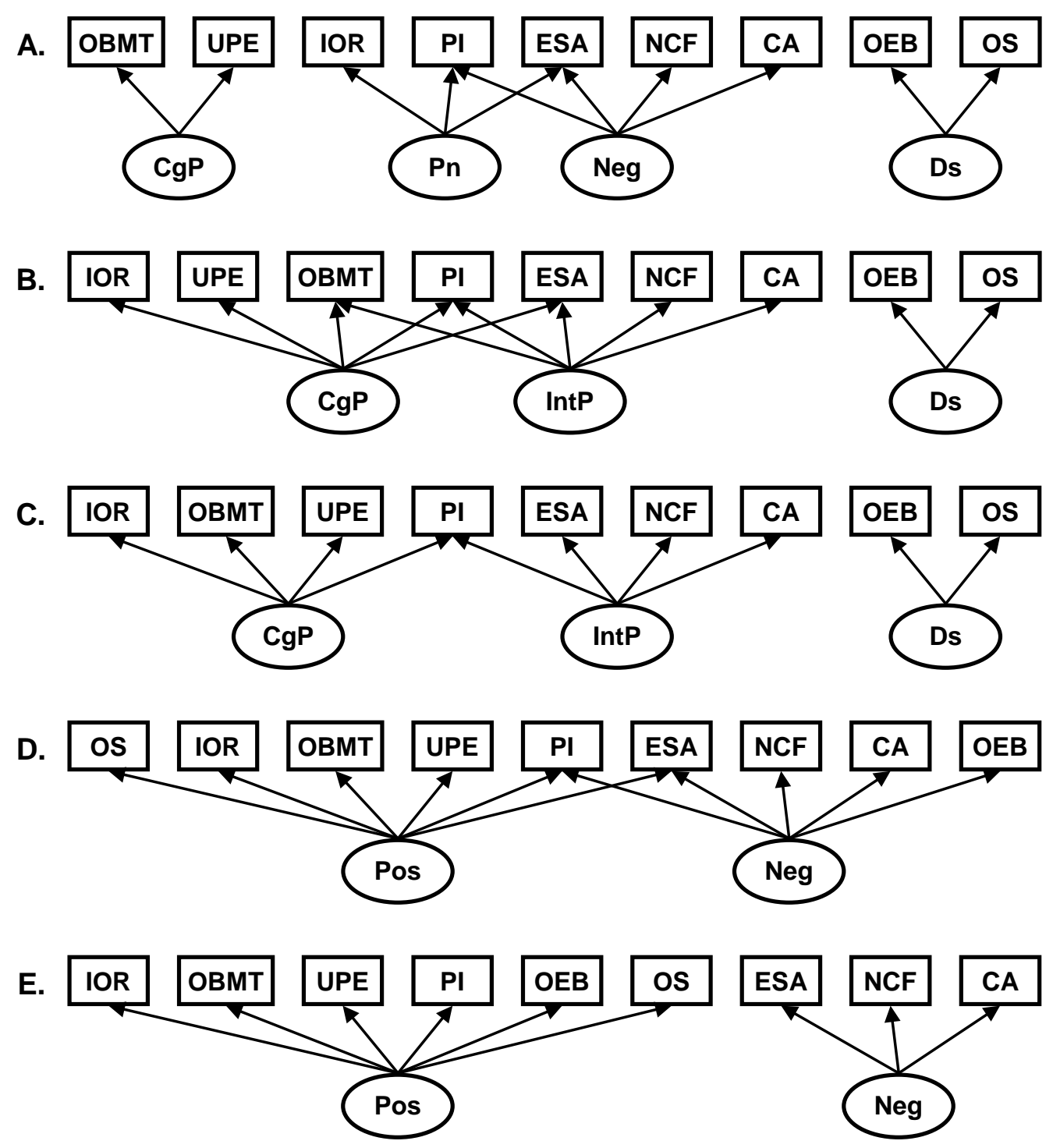

Figure 1. Five Measurement Models for the Nine SPQ Subscales. A. the 4-factor "paranoid" model of Stefanis et al. (2004); B. the modified 3-factor model of Wuthrich and Bates (2006); C. the standard 3-factor model of Raine et al. (2004); D. the 2-factor model based on conceptualizations of Kendler et al. (1991); E. the simple 2-factor model based on conceptualizations of Siever and Gunderson (1983). Factors are represented by ovals: $\mathrm{CgP}=$ cognitive-perceptual, $\mathrm{Pn}=$ paranoid, Neg=negative, Ds=disorganized, IntP=interpersonal, and Pos=positive. Subscales are represented by rectangles: OBMT=odd beliefs or magical thinking, UPE=unusual perceptual experiences, IOR=ideas of references, PI=paranoid ideation/suspiciousness, ESA=excessive social anxiety, NCF=no close friends, $\mathrm{CA}=$ constricted affect, $\mathrm{OEB}=$ odd or eccentric beliefs, and OS=odd speech. 